\title{
Combined locoregional and systemic therapy for advanced hepatocellular carcinoma: finally, the future is obscure
}

\author{
Khashayar Farsad ${ }^{1}$, Nima Nabavizadeh ${ }^{2}$, Adel Kardosh ${ }^{3}$, Janice H. Jou ${ }^{4}$, Willscott E. Naugler ${ }^{4}$, \\ Kenneth J. Kolbeck ${ }^{1}$ \\ ${ }^{1}$ Department of Interventional Radiology, Dotter Interventional Institute, Oregon Health and Science University, Portland, OR, USA; ${ }^{2}$ Department \\ of Radiation Oncology, Oregon Health and Science University, Portland, OR, USA; ${ }^{3}$ Division of Hematology and Medical Oncology, Department \\ of Internal Medicine, Oregon Health and Science University, Portland, OR, USA; ${ }^{4}$ Division of Gastroenterology and Hepatology, Department of \\ Internal Medicine, Oregon Health and Science University, Portland, OR, USA \\ Correspondence to: Khashayar Farsad. Dotter Department of Interventional Radiology, 3181 SW Sam Jackson Park Road, L-605, Portland, OR 97239, \\ USA. Email: farsad@ohsu.edu. \\ Comment on: Kim BK, Kim DY, Byun HK, et al. Efficacy and Safety of Liver-Directed Concurrent Chemoradiotherapy and Sequential Sorafenib for \\ Advanced Hepatocellular Carcinoma: A Prospective Phase 2 Trial. Int J Radiat Oncol Biol Phys 2020;107:106-15.
}

Submitted May 22, 2020. Accepted for publication Jun 12, 2020.

doi: 10.21037/atm-20-4164

View this article at: http://dx.doi.org/10.21037/atm-20-4164

Hepatocellular carcinoma (HCC) remains a devastating disease worldwide with a growing incidence and high mortality rate (1). Very few patients present with early stage disease that can be treated with curative intent with transplant, surgical resection, or in some cases, ablation. Intermediate stage patients are treated primarily with transarterial liver-directed therapies, including transarterial chemoembolization (TACE), chemoembolization with drug-eluting beads (DEB-TACE) or radioembolization with yttrium-90 (Y90), and advanced stage patients are primarily treated with systemic therapy or palliative care (2). Recently, growing experience and outcomes for external beam radiation therapy (XRT), particularly with the advent of $3 \mathrm{D}$ conformal techniques that reduce nontarget hepatotoxicity, have gained interest and have become recognized as a locoregional therapy for HCC (3-6). As the available armamentarium of agents has increased for use in HCC, there has been growing interest in combination therapies to maximally leverage the relative strengths of each therapy, and potentially to produce synergistic effects. How best to use the available treatments remains a topic of active research.

\section{Therapies for advanced HCC}

The landscape for HCC therapy has been evolving most rapidly for advanced disease. Particularly in the setting of central venous invasion, the somber outcomes for this disease have historically been minimally impacted by any therapy, if at all $(7,8)$. For over a decade, sorafenib has remained the sole agent showing benefit over placebo in advanced HCC, and that, just a modest increase in overall survival at best (7-9). In conjunction with the challenging side effect profile which reduces adherence in many patients, the limits of this therapy for advanced disease have opened extensive research for alternative agents. Newer therapies for advanced HCC have shown efficacy in the first or second line, and some with arguably improved patient tolerability compared with sorafenib. These include other multikinase inhibitors such as lenvatinib, regorafenib, and cabozantinib, as well as the vascular endothelial growth factor (VEGF) inhibitor, ramucirumab (10-13).

\section{Combination therapy and immunotherapy for HCC}

Given the inflammatory milieu of cirrhosis as a common background for HCC, research has increasingly examined whether immunotherapy may hold promise for therapeutic efficacy. Immunotherapy for HCC has focused on monoclonal antibodies blocking immune checkpoint inhibition pathways, primarily the programmed 
cell death pathway via the PD-1 receptor on T cells or its cognate ligand, PD-L1, on tumor cells and cells of the tumor microenvironment (14). Additionally, the immune checkpoint pathway mediated by the cytotoxic T-lymphocyte-associated antigen 4 (CTLA-4) receptor on $\mathrm{T}$ cells and it cognate ligand on antigen presenting cells has also been explored as a therapeutic target (14). A putative role for two PD-1 inhibitors, nivolumab and pembrolizumab, has been examined for efficacy in advanced HCC in the first or second line with encouraging early results $(15,16)$. Despite this, larger phase III trials using these agents as monotherapy failed to reach predefined statistically significant study endpoint targets, although some signals were noted showing putative improved differential responses in those getting immunotherapy $(17,18)$. Combination therapy with lenvatinib and pembrolizumab (Keynote-524) in the first line for Barcelona Clinic Liver Cancer (BCLC) stages $\mathrm{B}$ and $\mathrm{C}$ disease in patients ineligible for locoregional therapy showed promising early anti-tumor activity with good tolerance (19), and a phase 3 trial looking at this combination is currently underway (LEAP-002) (20). A recent phase 3 randomized controlled trial (IMBRAVE 150) using the anti-PD-L1 antibody, atezolizumab, plus the VEGF inhibitor, bevacizumab, compared to sorafenib has arguably been the most exciting representation of the relevance of immunotherapy for advanced HCC (21). Results from this trial showed that the median overall survival for the experimental arm was not yet reached at a median follow up interval of 8.6 months, with a $27.3 \%$ objective response rate (ORR) and moderately improved progression-free survival (PFS) (21). Importantly, the combination treatment was relatively well tolerated, with a similar incidence of adverse events compared to sorafenib, but with improvement in quality of life deterioration (21). The results from the IMBRAVE 150 trial have been so promising, there is new expectation this therapy may finally make a true dent in the lack of effective systemic therapy outcomes for advanced HCC seen thus far with so many prior agents. Furthermore, these experiences are showing efficacy for agents beyond multikinase inhibitors for demonstrable response rates in HCC.

All this is good, but it does serve to complicate matters. We have so many more therapeutic combinations at our disposal, and still so many ongoing trials promising newer results. The challenge will be how best to sift through the available data to arrive at a rational treatment algorithm that can benefit the most patients. Several trials are currently underway examining the role of combination therapies in
HCC (Table 1). Moreover, the positive outcomes from these newer agents have spurred several clinical trials pushing the use of combination and systemic therapies as first line in early and intermediate stage disease.

\section{Role of XRT for HCC}

So, where does XRT fall in this melee? External beam radiotherapy has been studied in every phase of HCC, with early stage therapy being explored as an alternative ablative modality, and its use in intermediate and advanced stages offered for palliative disease control $(3,4)$. More recently, XRT has been increasingly explored in combination therapy regimens. Combination XRT and TACE was shown in a phase 2 prospective study including 90 patients to improve outcomes for advanced HCC with portal venous invasion compared to sorafenib monotherapy (5). In addition, RTOG 1112 is further examining the role of combination therapy using stereotactic body radiotherapy (SBRT) and sorafenib.

More recently, Kim et al. presented a small-scale phase 2 clinical trial with 47 patients examining the role of upfront chemoradiation using XRT in combination with hepatic arterial chemoinfusion with 5 -fluorouracil (5-FU) and leucovorin via an implanted arterial pump, followed by systemic therapy with sorafenib (22). Nearly half (44.1\%) of the cohort presented with a solitary mass, potential rationale for a targeted approach upfront, and one third of the cohort (34\%) underwent synchronous TACE for lesions outside of the radiation treatment field. Radiation was provided over 20-25 fractions for a median dose of $60 \mathrm{~Gy}$ in the treatment bed. Median OS was 24.6 months for the cohort, and 13.0 months for the subgroup with main or first-branch portal vein invasion. By imaging, an ORR of $53.2 \%$ was achieved, with $44.7 \%$ ORR 4 weeks after completion of chemoradiation. Median PFS was 6.8 months, with the majority of disease progression occurring either in the liver outside the XRT treatment zone, or in distant metastases. The authors concluded that chemoradiation with maintenance sorafenib therapy provided favorable responses for advanced HCC (22).

The study by Kim et al., while provocative, leaves more questions than answers, presenting yet another twist on combination therapies for advanced HCC, this time using chemoradiation in combination with systemic therapy. This, of course, should not be surprising in the present HCC treatment landscape. As often the case when comparing results from Asia and Western regions, 
Table 1 Current trials for first-line combination therapy in HCC

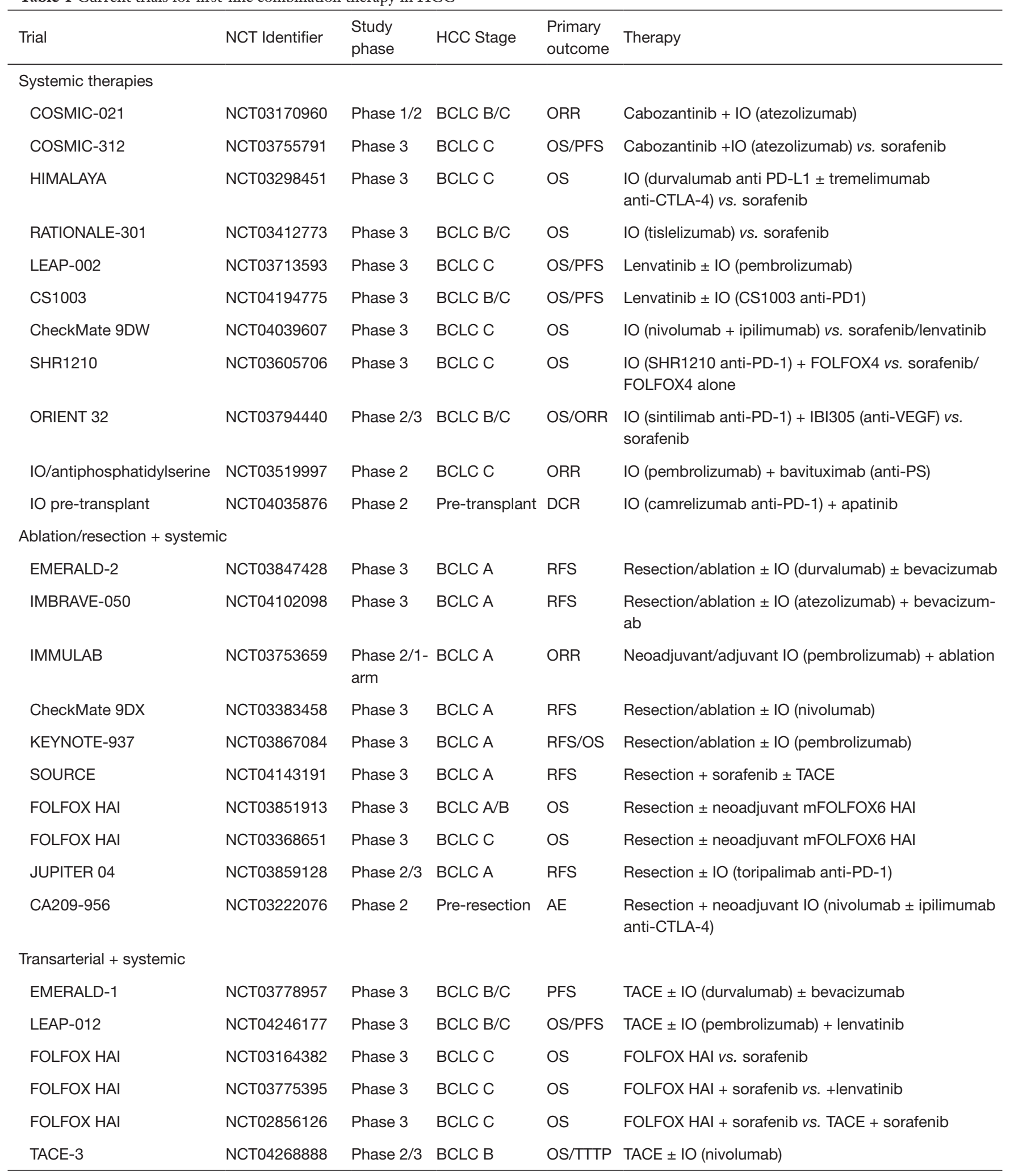

Table 1 (continued) 
Table 1 (continued)

\begin{tabular}{|c|c|c|c|c|c|}
\hline Trial & NCT Identifier & $\begin{array}{l}\text { Study } \\
\text { phase }\end{array}$ & HCC Stage & $\begin{array}{l}\text { Primary } \\
\text { outcome }\end{array}$ & Therapy \\
\hline CheckMate 74W & NCT04340193 & Phase 3 & BCLC B & OS/TTTP & TACE + IO (nivolumab + ipilimumab) vs. +nivolumab \\
\hline TAIPD1-HCC & NCT03869034 & Phase 2 & $\mathrm{BCLC} B / \mathrm{C}$ & PFS & FOLFOX HAI + IO (sintilimab) \\
\hline NASIR-HCC & NCT03380130 & Phase 1 & $B C L C B / C$ & $\mathrm{AE}$ & Y90 SIRT + IO (nivolumab) \\
\hline $\mathrm{TACE}+\mathrm{IO}$ & NCT03638141 & Phase 2 & BCLC B & ORR & TACE + IO (durvalumab + tremelimumab) \\
\hline TRIPLET & NCT04191889 & Phase 2 & BCLC C & ORR & FOLFOX HAI + IO (camrelizumab) + apatinib \\
\hline HCRN: Gl15-225 & NCT03099564 & Phase 1 & $\mathrm{BCLC} B / \mathrm{C}$ & PFS & Y90 SIRT + IO (pembrolizumab) \\
\hline \multicolumn{6}{|l|}{ XRT + systemic } \\
\hline \multicolumn{6}{|l|}{$\mathrm{XRT}+$ transarterial } \\
\hline SBRT + TACE & NCT03895359 & Phase 3 & BCLC A/B & OS/TTP & $\mathrm{TACE} \pm \mathrm{SBRT}$ \\
\hline TACE-EBRT & NCT03116984 & Phase 3 & $\mathrm{BCLC} B / \mathrm{C}$ & OS & TACE \pm XRT \\
\hline TACE-EBRT & NCT03939845 & Phase 3 & BCLC C & os & $\mathrm{TACE} \pm \mathrm{XRT}$ \\
\hline TACE-SBRT & NCT02794337 & Phase $2 / 3$ & BCLC B & TTP & TACE + XRT vs. TACE + sorafenib \\
\hline
\end{tabular}

HCC, hepatocellular carcinoma; BCLC, Barcelona Clinic Liver Cancer stage; IO, immunotherapy; ORR, objective response rate; PFS, progression-free survival; RFS, recurrence-free survival; DCR, disease control rate (proportion of patients with complete response, partial response, and stable disease); OS, overall survival; AE, adverse events; TTP, time to progression; XRT, external beam radiation therapy; SBRT, stereotactic body radiotherapy; TACE, transarterial chemoembolization; HAI, hepatic arterial infusion.

the underlying etiology of hepatitis B virus (HBV) as the driver of liver disease, and HCC, makes it difficult to generalize outcomes. As a dramatic example, nearly $20 \%$ of the patients in this cohort achieved definitive transplant or resection, even some with main portal vein tumor invasion (22). This phenomenon is effectively never seen in Western cohorts with primarily hepatitis $\mathrm{C}$ virus (HCV), alcohol or non-alcoholic steatohepatitis (NASH) related liver disease with the greater proportion of patients with HCC and concomitant cirrhosis. Furthermore, it is unclear whether outcomes were censored for transplant or resection, an effect which would artificially inflate the effect of the studied therapy. In addition, based on the treatment history of the patients on the trial, the combined outcomes are really reflective of multi-part therapy combining chemoinfusion, XRT, TACE, and systemic therapy with sorafenib. The heterogeneity of this experience, along with the heterogeneity of patient cohort, is relevant and may have significantly impacted the results, particularly when a control cohort treated with sorafenib alone was not studied. In many ways, this was a missed opportunity, although arguably not the goal of a phase II trial. That said, $27.7 \%$ of the cohort ultimately did not receive sequential sorafenib therapy, and $10.6 \%$ were rendered ineligible due to toxicity from XRT, further impacting a clear understanding of outcomes (22). In regard to the choice of treatments on trial, experience with chemoradiation and chemoinfusion via an implantable pump for HCC has been limited outside of Asia, has had conflicting results, and may not be routinely available. Furthermore, chemoradiation with hypofractionation has not been rigorously compared to other radiation delivery techniques such as SBRT, which has gained favor for HCC in North America (6). Additionally, sorafenib has been shown less effective against HBV-related HCC (7), leaving open the question of whether even better outcomes on trial may have been observed with different 
maintenance systemic therapy regimens. Nevertheless, this study contributes to the mounting interest in combining XRT and systemic therapy, both with existing molecular targeted agents and with immunotherapy. It is therefore important to carefully interpret the results of this study within the larger context of current trials underway for advanced HCC.

\section{Combination locoregional and systemic therapy for HCC}

Combination therapies for HCC are increasingly being seen to provide superior treatment responses compared to monotherapies. This has been observed with TACE and ablation in early stage disease and is now being considered more fervently in intermediate and advanced stage disease. Although in the adjuvant setting, addition of sorafenib historically has not shown superiority over monotherapy with TACE or resection/ablation $(23,24)$, current phase III trials are underway examining the role of newer systemic therapies with resection or ablation in early stage HCC, and with TACE for intermediate stage disease (Table 1). Both strategies include immune checkpoint inhibition as part of the treatment paradigm, with the idea that tumor antigens may be released as part of locoregional therapy to augment the efficacy of immunotherapy. More aggressive combination therapies for advanced HCC have also been explored, with several including combination systemic therapies, but also combination locoregional and systemic therapies (Table 1). In this realm, locoregional radiotherapies are intriguing, as the potential immunogenicity of radiotherapy has been proposed as a rationale for combination therapies with immune checkpoint inhibition (25). From the perspective of transarterial therapy, the small (30-60 micron) particles used in radioembolization are less likely to pose ischemic risks in the setting of portal venous invasion with advanced HCC (26). With external beam radiotherapy, the concept of treatment tolerability and ability to target suitable radiation fields with portal venous invasion is of interest $(6,27)$. Considering the majority of patients presenting with advanced HCC nevertheless have liver-only or liver-dominant disease, there is appeal in a combination approach that not only delivers maximum potential therapy to the site of greatest tumor burden, but also provides additional putative durability of treatment response by way of systemic therapy given the high-risk profile for rapid disease progression.

\section{Concluding remarks}

Despite the four decades during which locoregional therapies have been used for HCC, primarily by way of TACE, it is notable that it has only been in the last twenty years that we have shown through rigorous trials that this therapy is superior to best supportive care. Over the last decade, treatment of HCC has arguably been without much controversy: transplantation or resection for those few who were candidates, ablation for the majority with early stage disease who were not surgical candidates, TACE or radioembolization for intermediate stage disease, and sorafenib or palliative care for those with advanced disease. It has really been in the last few years where we have seen the emergence of newer therapeutics, and more aggressive combination therapies, that we have increased the potential for actually changing outcomes for this grave disease. As such, for patients other than those with early stage disease where transplantation or resection remain the best chance for a durable outcome, it has become very challenging now to know what the optimum therapies are. This obfuscation, while potentially frustrating, could be welcomed because now we truly have more options on the table. In many ways, the increasing heterogeneity of treatments for HCC mimics the high degree of molecular heterogeneity in HCC itself. Improved molecular diagnostics of patient tumors by way of increasing biopsy banks will therefore be crucial to better identify those most likely to benefit from the increasing availability of therapeutic options. Furthermore, big data analytical tools, including omics-based approaches, will undoubtedly be needed to help sift through the heterogeneity of patient treatments and outcomes. To invoke the infamous quote by Mark Twain, "If you don't like the weather in New England now, just wait a minute." Such is the current climate of HCC therapies, and it will be incumbent on all of us to keep our eyes on the horizon.

\section{Acknowledgments}

Funding: None.

\section{Footnote}

Provenance and Peer Review: This article was commissioned by the editorial office, Annals of Translational Medicine. The article did not undergo external peer review.

Conflicts of Interest: The authors have completed the 
ICMJE uniform disclosure form (available at http://dx.doi. org/10.21037/atm-20-4164). KF reports other from Auxetics, Inc., personal fees from Cook Medical, personal fees from BTG, personal fees from Neuwave, grants and personal fees from Guerbet, LLC, personal fees from Genentech, personal fees from Dova Pharmaceuticals, outside the submitted work; In addition, KF has a patent "use of specific stent class for the management of venous stenosis" licensed to Auxetics, Inc. The other authors have no conflicts of interest to declare.

Ethical Statement: The authors are accountable for all aspects of the work in ensuring that questions related to the accuracy or integrity of any part of the work are appropriately investigated and resolved.

Open Access Statement: This is an Open Access article distributed in accordance with the Creative Commons Attribution-NonCommercial-NoDerivs 4.0 International License (CC BY-NC-ND 4.0), which permits the noncommercial replication and distribution of the article with the strict proviso that no changes or edits are made and the original work is properly cited (including links to both the formal publication through the relevant DOI and the license). See: https://creativecommons.org/licenses/by-nc-nd/4.0/.

\section{References}

1. Yang JD, Hainaut P, Gores GJ, et al. A global view of hepatocellular carcinoma: trends, risk, prevention and management. Nat Rev Gastroenterol Hepatol 2019;16:589-604.

2. Forner A, Reig M, Bruix J. Hepatocellular carcinoma. Lancet 2018;391:1301-14.

3. Bujold A, Massey CA, Kim JJ, et al. Sequential phase I and II trials of stereotactic body radiotherapy for locally advanced hepatocellular carcinoma. J Clin Oncol 2013;31:1631-9.

4. McPartlin AJ, Dawson LA. Stereotactic Body Radiotherapy for Hepatocellular Carcinoma. Cancer J 2016;22:296-301.

5. Yoon SM, Ryoo BY, Lee SJ, et al. Efficacy and Safety of Transarterial Chemoembolization Plus External Beam Radiotherapy vs Sorafenib in Hepatocellular Carcinoma With Macroscopic Vascular Invasion: A Randomized Clinical Trial. JAMA Oncol 2018;4:661-9.

6. Nabavizadeh N, Waller JG, Fain R, 3rd, et al. Safety and Efficacy of Accelerated Hypofractionation and Stereotactic Body Radiation Therapy for Hepatocellular Carcinoma
Patients With Varying Degrees of Hepatic Impairment. Int J Radiat Oncol Biol Phys 2018;100:577-85.

7. Cheng AL, Kang YK, Chen Z, et al. Efficacy and safety of sorafenib in patients in the Asia-Pacific region with advanced hepatocellular carcinoma: a phase III randomised, double-blind, placebo-controlled trial. Lancet Oncol 2009;10:25-34.

8. Llovet JM, Ricci S, Mazzaferro V, et al. Sorafenib in advanced hepatocellular carcinoma. N Engl J Med 2008;359:378-90.

9. Bruix J, da Fonseca LG, Reig M. Insights into the success and failure of systemic therapy for hepatocellular carcinoma. Nat Rev Gastroenterol Hepatol 2019;16:617-30.

10. Abou-Alfa GK, Meyer T, Cheng AL, et al. Cabozantinib in Patients with Advanced and Progressing Hepatocellular Carcinoma. N Engl J Med 2018;379:54-63.

11. Bruix J, Qin S, Merle P, et al. Regorafenib for patients with hepatocellular carcinoma who progressed on sorafenib treatment (RESORCE): a randomised, double-blind, placebo-controlled, phase 3 trial. Lancet 2017;389:56-66.

12. Kudo M, Finn RS, Qin S, et al. Lenvatinib versus sorafenib in first-line treatment of patients with unresectable hepatocellular carcinoma: a randomised phase 3 noninferiority trial. Lancet 2018;391:1163-73.

13. Zhu AX, Kang YK, Yen CJ, et al. Ramucirumab after sorafenib in patients with advanced hepatocellular carcinoma and increased alpha-fetoprotein concentrations (REACH-2): a randomised, double-blind, placebocontrolled, phase 3 trial. Lancet Oncol 2019;20:282-96.

14. Kudo M. Immuno-Oncology Therapy for Hepatocellular Carcinoma: Current Status and Ongoing Trials. Liver Cancer 2019;8:221-38.

15. El-Khoueiry AB, Sangro B, Yau T, et al. Nivolumab in patients with advanced hepatocellular carcinoma (CheckMate 040): an open-label, non-comparative, phase 1/2 dose escalation and expansion trial. Lancet 2017;389:2492-502.

16. Zhu AX, Finn RS, Edeline J, et al. Pembrolizumab in patients with advanced hepatocellular carcinoma previously treated with sorafenib (KEYNOTE-224): a non-randomised, open-label phase 2 trial. Lancet Oncol 2018;19:940-52.

17. Finn RS, Ryoo BY, Merle P, et al. Pembrolizumab As Second-Line Therapy in Patients With Advanced Hepatocellular Carcinoma in KEYNOTE-240: A Randomized, Double-Blind, Phase III Trial. J Clin Oncol 2020;38:193-202. 
18. Yau T, Park JW, Finn RS, et al. CheckMate 459: A randomized, multi-center phase III study of nivolumab (NIVO) vs sorafenib (SOR) as first-line (1L) treatment in patients (pts) with advanced hepatocellular carcinoma (aHCC). Ann Oncol 2019;30:v851-v934.

19. Ikeda M, Sung MW, Kudo M, et al. Abstract CT061: A Phase Ib trial of lenvatinib (LEN) plus pembrolizumab (PEMBRO) in unresectable hepatocellular carcinoma (uHCC): Updated results. Ann Oncol 2019;30:v253-v324.

20. Llovet JM, Kudo M, Cheng AL, et al. Lenvatinib (len) plus pembrolizumab (pembro) for the first-line treatment of patients (pts) with advanced hepatocellular carcinoma (HCC): Phase 3 LEAP-002 study. J Clin Oncol 2019;37. doi: 10.1200/JCO.2019.37.15_suppl.TPS4152.

21. Finn RS, Qin S, Ikeda M, et al. Atezolizumab plus Bevacizumab in Unresectable Hepatocellular Carcinoma. N Engl J Med 2020;382:1894-905.

22. Kim BK, Kim DY, Byun HK, et al. Efficacy and Safety of Liver-Directed Concurrent Chemoradiotherapy and Sequential Sorafenib for Advanced Hepatocellular Carcinoma: A Prospective Phase 2 Trial. Int J Radiat

Cite this article as: Farsad K, Nabavizadeh N, Kardosh A, Jou JH, Naugler WE, Kolbeck KJ. Combined locoregional and systemic therapy for advanced hepatocellular carcinoma: finally, the future is obscure. Ann Transl Med 2020;8(24):1700. doi: 10.21037/atm-20-4164
Oncol Biol Phys 2020;107:106-15.

23. Bruix J, Takayama T, Mazzaferro V, et al. Adjuvant sorafenib for hepatocellular carcinoma after resection or ablation (STORM): a phase 3, randomised, double-blind, placebo-controlled trial. Lancet Oncol 2015;16:1344-54.

24. Lencioni R, Llovet JM, Han G, et al. Sorafenib or placebo plus TACE with doxorubicin-eluting beads for intermediate stage HCC: The SPACE trial. J Hepatol 2016;64:1090-8.

25. Choi C, Yoo GS, Cho WK, et al. Optimizing radiotherapy with immune checkpoint blockade in hepatocellular carcinoma. World J Gastroenterol 2019;25:2416-29.

26. Zu Q, Schenning RC, Jahangiri Y, et al. Yttrium-90 Radioembolization for BCLC Stage C Hepatocellular Carcinoma Comparing Child-Pugh A Versus B7 Patients: Are the Outcomes Equivalent? Cardiovasc Intervent Radiol 2020;43:721-31.

27. Shui $Y, Y u$, Ren X, et al. Stereotactic body radiotherapy based treatment for hepatocellular carcinoma with extensive portal vein tumor thrombosis. Radiat Oncol 2018;13:188. 